\title{
MEASURING DRIVER BEHAVIOUR - INDICATORS FOR TRAFFIC SAFETY
}

\author{
Michał Niezgoda, Tomasz Kamiński, Mikołaj Kruszewski \\ Motor Transport Institute \\ Transport Telematics and Management Centre \\ Jagiellonska Street 80, 03-301 Warsaw, Poland \\ tel.: +4822 8113231, fax: +48228113231 \\ e-mail:michal.niezgoda@its.waw.pl \\ tomasz.kaminski@its.waw.pl,mikolaj.kruszewski@its.waw.pl
}

\begin{abstract}
Over one million people die each year on the roads and about 20-50 million are injured. Traffic safety is a global problem that needs interdisciplinary scientific approach to develop methodology for safety studies that will lead to valid conclusions and in the end to suitable preventive measures. It is difficult to define what a safe and unsafe behaviour is. Transportation research tries to find relation between significant variables and traffic safety in the triangle of interaction between human, vehicle and environment. Raw accidents data excludes ex-ante evaluations and is insufficient due to the rare frequency and difficulty with investigating causes. There is still lack of consensus between researchers on usage of the traffic safety indicators and their cut-off values. Article presents different methods for measuring driver behaviour and objective behavioural indicators that are commonly used as traffic safety measures e.g. time to collision, post-encroachment-time, deceleration rate, time headway, red-light violations, lane deviations, gap acceptance and others. The aim of the paper is to provide general framework for exploring valid and reliable variables in the traffic safety studies.
\end{abstract}

Keywords: traffic safety indicators, driver behaviour, accidents

\section{Introduction - methods to measure driver behaviour}

World Health Organization in the global status report on road safety estimates that road traffic injuries are $9^{\text {th }}$ leading cause of death. However, in 2030 WHO predicts that in 2030 it is going to be $5^{\text {th }}$ even before HIV/AIDS. Road traffic is a dynamic system dependant on the regulated relations between users (e.g. pedestrians, drivers), vehicles and environment (infrastructure). This is still a very simplified definition. It is a complex system with almost seamless number of variables, which can influence the probability of causing accidents. Today safety is one of the key challenges for transportation research. Road safety science tries to address this issue with an interdisciplinary approach that uses different theories and statistical methods to find significant factors that are relevant to traffic safety. Svensson [18] points out that it is possible to operationalise and find link between different approaches to traffic safety theories - both psychological and engineering e.g. by analyzing relationship between severity hierarchy of traffic events and behaviour of drivers. Nevertheless, there is a broad scientific consensus that most of the traffic accidents are due to human error. Quantifying human factors that influence traffic safety requires valid and reliable indicators. There is a growing need for interdisciplinary standards that are used in measuring driver behaviour and performance.

There are different study methods that can be used for measuring driver behaviour e.g. selfreported/ expert-reported behaviours, vehicle measures of driving performance and even psychophysiological measures. Despite the fact that self-reported measures of driving are subjective, they provide interesting insight into the factors related with accidents involvement. Among most popular self-reported measures of driving behaviour are Driver Behaviour Questionnaire (DBQ), Driving Quality Scale (DQS), Driving Skill Inventory (DSI), Driver Anger Scale and Driver Attitude Questionnaire (DAQ). Undoubtedly, DBQ is most widely used questionnaire in the world. It has 
also many versions with different number of items (10-102). It is based on the human error theory of James Reason, which locates accident behaviours in the error-violation framework. Reason's questionnaire in its first version consists of 50 questions about driver's propensity to commit different aberrant behaviours. By conducting Principal Component Analysis on the sample of questioned drivers, Reason $[15,16]$ extracted two basic structures - violations, and errors. Error is defined as an event where planned sequence of mental or physical activity fail to achieve intended outcome and violations are rather deliberate deviations from those practices deemed necessary to maintain safe operation of the potential hazardous system. Errors and violations are complex behaviours that are mediated by different psychological mechanisms. e.g. errors are more due to cognitive failures and violations are strongly dependant on social context. Some authors indicate that correlation between DBQ factors and reported accidents may be in the region of .1-.4. Driving Quality Scale (DQS) is a measure of how well the person thinks he or she has driven (1-100, I drove extremely badly to +100 I drove extremely well). Other popular scale DAQ (Driver Attitude Questionnaire) provides measurement of respondent's attitudes towards four major driving issues - drink driving, following closely to other vehicles, risky overtaking and speeding. However, self-reported data is quite sensitive to many biases e.g. overestimation of our skills, social desirability and consistency motif. Correlation between self-reported accidents and DBQ behaviours can be driven by tendency to fill in questionnaire items in congruent manner [4].

Another way of studying driver behaviour is to use expert-reported measures of driving performance. These kinds of measures can be compared to real driving tests, where trained experts fill ready-made prospects with defined items relevant to traffic safety and driving performance. Experts make an observation and judge user's behaviour according to given situations. One of the most commonly used method is standardized Vienna Driving Test - "Wiener Fahrprobe" [2]. This test was developed during years of research work on traffic safety and used in many European traffic safety projects. Austrian method is based on observation of driver errors and his interaction with different road users in the real driving environment. Driver is accompanied by two observers - free observer without standardized observation sheet, who registers all kind of erroneous behaviour representing a sever offence of the law and causing danger - coding observer who has to describe the behaviour of the driver along all sections of the predefined test route using a standardized coding system (e.g. choice of lane on multi-lane roads). Authors [2] found statistically significant correlations between coded variables and accident circumstances registered by the police (between .38-.48). Very often in export-reported measures observers make a global evaluation of the driver's behaviour. This global assessment is a useful addition to the detailed and standardized evaluation.

Self-reported and expert-report usually consists of complex and difficult to define measures, which are also more or less subjective. Unbiased raw data can be recorded directly from the vehicle or even the driver. Vision is considered the most valuable source of information for the driver [7]. Our eyes can move even three times per second. Aspects of visual attention selectivity and eye movements can be measured with the eye-tracking technique. There also exist other physiological measures. Electroencephalogram (EEG) records electrical activity of the brain and is a typical measure of low vigilance state. Galvanic skin resistance (GSR) and electrodermal activity (EDA) are common techniques for defining stress level and workload. Measuring changes in the inter-beat-intervals with electrocardiogram (ECG) can be also a valid indicator of stress level. Also vehicle with dedicated devices can become a reliable source of information e.g. speed, rpm, steering wheel, pedals, gears, other controls or even distance to the another vehicle. These all measures proofed to be valid behavioural indicators of road safety.

\section{Behavioural and proximal indicators of traffic safety}

In many studies accident statistics are used to predict level of road safety and evaluate applied measures. However, very often due to underreporting and problem with identifying causes this type 
of data is inaccurate and unreliable (especially at the national level). Recording just traffic accidents, which are in their nature very rare events, excludes ex-ante evaluations and preventive measures [10]. Moreover, from the transport-engineering point of view traffic is a set of different microscopic events - not only accidents. This traffic process can be seen as a continuum of undisturbed passages, potential conflicts, slight conflicts, near accidents and accidents [8]. These assumptions are fundamental for Traffic Conflict Techniques used in both Europe, and United States that are aimed at detecting and counting "near accident" events that occur in traffic situations. Standardized definition of a traffic conflict was agreed as "a conflict consists in an interaction between two different road-users (or between one road user and road environment) that would shortly lead to collision unless one at least of the road users involved performed an immediate evasive action" [11]. Operational description of conflict is differs between researchers; however it is very often based on objective measurements (e.g. TA - time to accident). Some researchers argue validity of accident data and state that some safety indicators can better represent characteristic of unsafe interactions than raw accident figures [1]. These safety indicators can be seen as a reasonable statistical basis for different traffic models and theories.

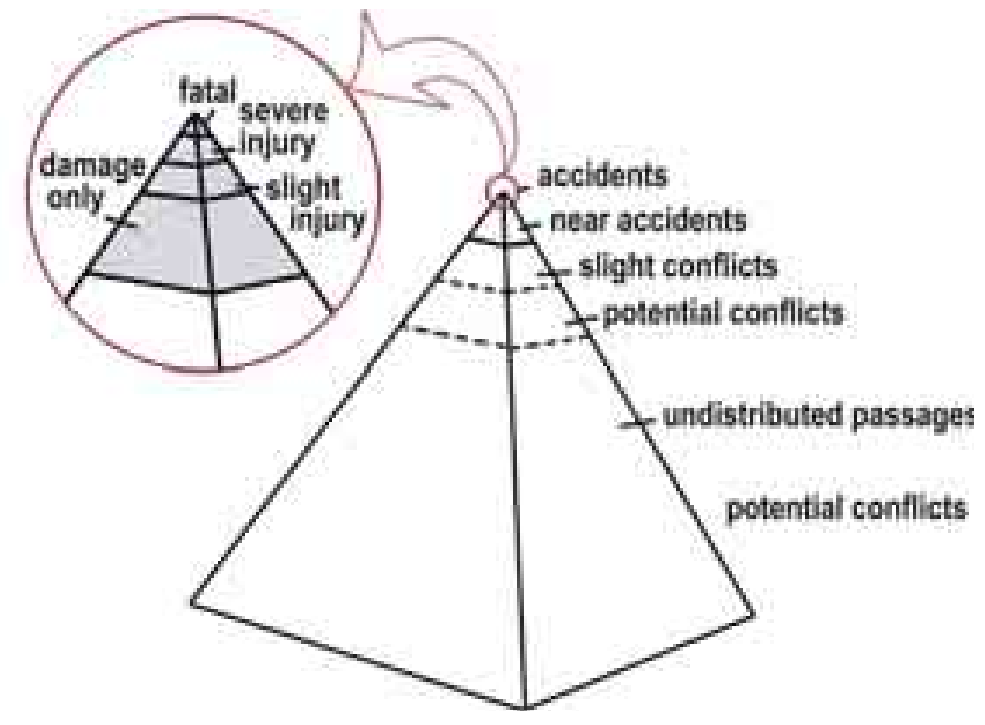

Fig. 1. The continuum of traffic events from undisturbed passages to fatal accidents [8]

Set of commonly used indicators in road traffic studies are referred as "proximal indicators". These proximal indicators can be associated with abovementioned traffic conflicts. This term comes with a definition of accident proximity in terms of time and spatial measures. It defines closeness of other vehicles or road users in relation to possible point of collision. The main advantage of proximal indicator comparing to accident measure is frequency of occurring. This measure is more proactive and provides more statistically reliable results. Particular proximal indicator and its measure depend on specific concept or methodology. Commonly used is e.g. Traffic Conflict Technique (TCT), Time to Accident (TA), Time to Collision (TTC) and Post Encroachment Time (PET). Svensson [18] proposed four criteria to define useful indicators of traffic safety that can be refereed as proximal. They have to: 1) be complementary to the accident figures and be more frequent than crashes, 2) be observable in the traffic 3) have not only statistically significant relationship to accidents but clear casual relation (be prior to the event and be part of the situational process), 4) have the characteristic of continuum of traffic events that are near-accidents. This last criterion separates other well-proofed traffic measures of safety that are used in transportation studies e.g. mean speed, gap-acceptance, time headway between vehicles, frequency of red-light violations and major lane deviations. All of the abovementioned proximal and non-proximal measures can be referred as behavioural indicators of traffic safety. These indicators are proofed to be valid at macro level, however to be able to refer them to micro level (individual) we need to take into consideration relation to psychological tests and measures. 


\section{Proximal indicators of traffic safety}

One of the most well known proximal indicators of traffic safety is Time-to-Collision and it is defined as the time that remains until a collision between two vehicles would have occurred if the collision course and speed difference were maintained [1]. It can be assumed that TTC occurs

Tab. 1. The number of events an average driver faces in the traffic [18]

\begin{tabular}{|l|l|l|}
\hline \multicolumn{1}{|c|}{ Event } & \multicolumn{1}{|c|}{ Frequency (time) } & \multicolumn{1}{c|}{ Frequency (travelled distance) } \\
\hline Traffic information & 5 in 1 second & 300 per 1 kilometre \\
\hline Driver observations & 2 in 1 second & 120 per 1 kilometre \\
\hline Driver decisions & 40 in 1 minute & 40 per 1 kilometre \\
\hline Driver actions & 30 in 1 minute & 30 per 1 kilometre \\
\hline Driver errors & 1 in 2 minutes & 1 er 2 kilometres \\
\hline Risky situations & 1 in 2 hours & 1 per 120 kilometres \\
\hline Near-accidents & 1 in 1 month & 1 per 2000 kilometres \\
\hline Accidents & 1 in 7.5 years & 1 per 150000 kilometres \\
\hline Injury accidents & 1 in 100 years & 1 per 2000000 kilometres \\
\hline Fatal accident & 1 in 2000 years & 1 per 40000000 kilometres \\
\hline
\end{tabular}

(value is finite) only in the case of higher speed of the following vehicle comparing to the speed of the lead vehicle. This assumption implies occurrence of collision course. Studies use recorded minimum value of Time-to-Collision of relevant events, value when evasive action started or duration of the defined TTC event. Relation between Time Headway and Time-To-Collision implies that low value of TTC is impossible for vehicles with high value of TH, however opposite situation is not true. Short Time Headway does not mean short Time-to-Collision. It means that short TH can be sustained for a long period of time where short TTC is not possible to sustain without causing a collision. This statement has consequences for traffic safety measurement, it is suggested that TTC is more relevant for occurrence of possible near-accident events. There is still no consensus among researchers about critical value of TTC; however it is proposed for safety studies that this value should be no less than 1.5 second and no more than 5 seconds [10].

Raw TTC measure can be considered as not valid in all critical situations. TTC value is calculated from speed and distance and it can give the same value for $20 \mathrm{~km} / \mathrm{h}$ and $160 \mathrm{~km} / \mathrm{h}$. To distinguish severity of these non-equal situations TTC can be combined with other measures e.g. braking rate or absolute value of speed at defined TTC event. There also exist extended measures of TTC - e.g. Time Exposed TTC (length of event time) and Time Integrated TTC (measure of TTC profile below the cut-off value). However, TTC measures does not describe relation between vehicle and vulnerable users e.g. pedestrians. Another variation of TTC measure is Time-to-Zebra (TTZ), which purpose is to estimate traffic safety at pedestrian's crossings and analyze if drivers regard pedestrians not on the crossing as a potential danger risk. TTZ value is defined as the distance to the zebra crossing divided by the speed [20]. It can be calculated as the time left for the car to the zebra crossing at the moment the pedestrian arrives at the curb. However, for TTZ determining start of the conflict is more complex than for TTC.

Other commonly used value is Post-Encroachment-Time (PET). This is a different measure that TTC because it excludes a collision course criterion. It is a calculation of time difference between the passages of two road users with a common area of potential collision (common spatial point) that is below a given cut-off value. This indicator includes fixed projected point of collision instead of speed and distance measures; however it is difficult to define severity of PET event without the speed value. It is also possible to other derivatives of PET measure e.g. gap time, encroachment time or initially attempted post encroachment time. Federal Highway Administration proposes other measures characterizing proximal indicators (conflict measures) which are deceleration rate and proportion of stopping distance. Deceleration rate is a rate at which crossing vehicle must decelerate to avoid collision. Deceleration-to-Safety Time is characterized, as necessary deceleration 
to reach the non-negative Post-Encroachment-Time of the movements of the conflict vehicle remains unchanged.

\section{Other behavioural indicators of traffic safety}

Within the driving simulation method, many different measures can be used. Driving speed is very common parameter related to accident risk. Speed affects traffic safety in two main ways. Higher

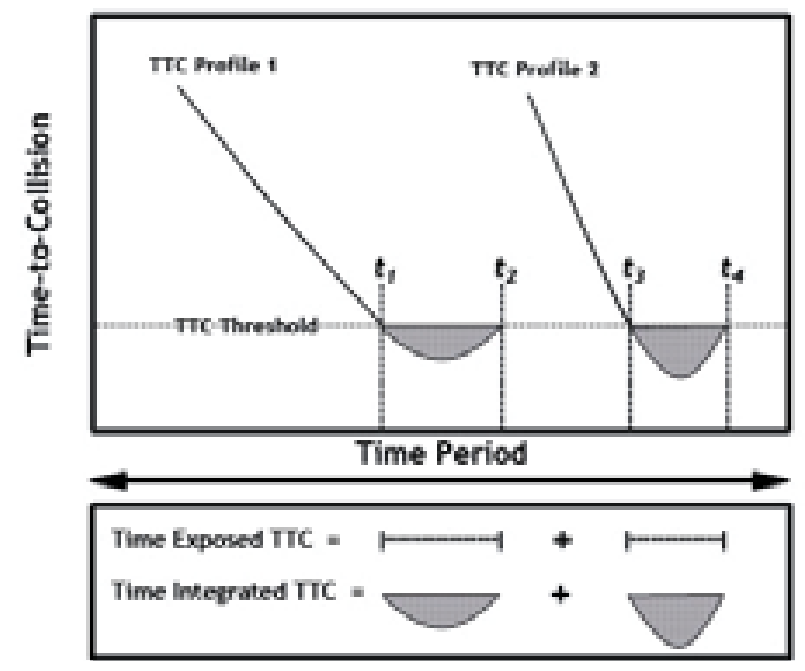

Fig. 2. TTC profiles and two different extended TTC measures - Time Exposed TTC and Time Integrated TTC [1]

speed gives driver less time to react in proper way to potentially hazardous situations that can occur on the road. It also gives other road actors (e.g. vulnerable road users) less time to react. Second type of influence is associated with kinetic energy that increases with speed and causes risk of fatal or severe accident. According to several authorities e.g. National Highway Traffic Safety Administration [12], exceeding the speed limit or driving too fast for road conditions (speeding) is one of the most prevalent factors contributing to traffic crashes, and a contributing factor in nearly $3 / 10$ fatal crashes. This relationship inspired the work on the speed adaptation systems, which have shown positive trend in relation to improving traffic safety. However, there are still many controversies and critical reviews on relationship between speed and traffic accidents.

Choice of speed is a result of a number of human factors and their complex interactions e.g. risk of apprehension and attitudes. Situational factors can influence our speed also, e.g. drivers mean speed decreases with increased workload. There are other non-psychological factors that can influence our speed choice e.g. roadway characteristic, posted speed limits, time of the day, risk of being ticketed, weather and visibility conditions. Moreover, from the point of view of questionnaires speed as a variable is difficult to measure. There are differences between self-reported speed and observed speed, these correlations vary between .27 to .65 [5]. That is the reason why speed needs objective criteria. Speed can be measured in the standardized observation e.g. above/below the speed limit, unsteady speed, abruptly braking or in the relation to speed of other vehicles. However, most widely used variable is mean speed.

There are several well-known safety predictive models based on the relationship between speed and accidents. According to the Power Model of Nilsson, the effects of changes in speed on the number of accidents and the severity of injuries can be estimated by means of a set of power functions. Swedish research by Nilsson was based on the empirical data from a large number of speed limit changes in Sweden and assessing the changes in the accidents number and their severity due to the changes in mean speeds on the selected sections. However, this power model was prepared on the basis of empirical data from predominantly higher speed limit zones. Due to more recent research work [3] Nilsson's power model and influence of average speed on crash risk is more adequate on rural areas (highways) than on urban roads and lower speed zones. 
Nilsson power model can be summarized in terms of six equations (1-6) that relate changes in the number of accidents and victims to changes in the mean speed of traffic. Speed is denoted by $V$, accidents by $Y$ and victims by $Z$ [3]:

- number of fatal accidents:

$$
Y_{1}=\left(\frac{V_{1}}{V_{0}}\right)^{4} Y_{0}
$$

- number of fatalities:

$$
Z_{1}=\left(\frac{V_{1}}{V_{0}}\right)^{4} Y_{0}+\left(\frac{V_{1}}{V_{0}}\right)^{8}\left(Z_{0}-Y_{0}\right)
$$

- number of fatal and serious injury accidents:

$$
Y_{1}=\left(\frac{V_{1}}{V_{0}}\right)^{3} Y_{0},
$$

- number of fatal or serious injuries:

$$
Z_{1}=\left(\frac{V_{1}}{V_{0}}\right)^{3} Y_{0}+\left(\frac{V_{1}}{V_{0}}\right)^{6}\left(Z_{0}-Y_{0}\right),
$$

- number of injury accidents:

$$
Y_{1}=\left(\frac{V_{1}}{V_{0}}\right)^{2} Y_{0}
$$

- number of injured road users (all):

$$
Z_{1}=\left(\frac{V_{1}}{V_{0}}\right)^{2} Y_{0}+\left(\frac{V_{1}}{V_{0}}\right)^{4}\left(Z_{0}-Y_{0}\right) .
$$

Single mean speed parameter may be not sufficient. There are studies stating that variance of speeds and relation of drivers speed to speed of other vehicles needs to be taken into consideration. One of the most controversial and famous findings was U-shaped curve of Salomon [9] that found different and infamous relation between speed and accidents involvement. This curve can be described as a function of speeds of other vehicles with speeds around the median speed, which is a speed with lowest probability of accident involvement. In other words crash risk is higher if the driver has greater difference between his own speed and the average speed of traffic flow. It led to infamous belief among some group of traffic engineers that it is more important to get similar speed for vehicles than to reduce average speed of traffic. However, today methodology of Salomon studies is being questioned. Other studies [21] tried to find correlation between traffic accidents and other variables that can be classified as longitudinal and lateral control - g-force that drivers experience when vehicle turns brakes or accelerates. However, these pilot studies showed only weak tendency for one variable of g-force (Right/Left) that predicts drivers' accidents involvement.

Gap-acceptance is considered as one of the most important determinants of traffic safety at intersections. There is a number of different approaches to the scientific investigation and modelling of this behavioural indicator. Gap-acceptance is characterized as the interval between following vehicles in the stream of traffic flow, which driver accepting as the one when he could join or cross the stream. In many statistical models it could be used also the 'critical gap' indicator, which is the smallest accepted gap. Some research suggests that gap-acceptance is not consistent over time (i.e. [13]) and could be related to the risk-reward loop process, which seems to be in line with real-world situations. There is a number of factors which were proved to affect gap-acceptance process, which are i.e.: age, gender, the existence of passengers in the vehicle, time spent waiting at the head of a queue, traffic flow and type of vehicles in the primary traffic stream. 
Other important factor influencing traffic safety is called Time Headway (TH). Time Headway describes the time, in seconds, between two successive vehicles as they pass a point on the roadway, measured from the same common feature of both vehicles [6]. Headway is used basically in measures and research concerning uninterrupted flow i.e. at the highway. Headway is used not only as a traffic safety indicator, but also as a variable used for traffic streams measures in transportation engineering discipline. Safe headway differs between countries. Most reliable relations between traffic safety and headway value concerns only critical headway, which is the threshold between safe and unsafe driving, but there are still some variances between critical headway value in different approaches (the values between 1.0-1.5 seconds are most often cited in literature).

Next traffic safety indicator is closely related with main causes of accidents at signalized intersections and is described as "red-light violations" indicator. Most research proves that redlight violations are mainly the result of driver impatience, poor speed adaptation, and lack of judgement, but some have correlated it also with personality treats such as thrill-seeking [14]. However very important red-light violation factor is very difficult to measure in terms of personal safety indicator, and is basically used only in system approach.

Among traffic safety indicators were also described some indicators concerning lateral behaviour of a driver. The most popular are mean lane position, standard deviation of lane position, lane exceed and Time-To-Line-Crossing [10]. Lateral behaviour, sometimes called also 'lane keeping', describes driving performance, but it is very difficult to indicate where limitations between safety and unsafe behaviours are. Related to this, it is likely that the experienced drivers do not continuously pursue some precise lane position, but seeks to maintain the vehicle within some satisfactory range. At the same time increased lane swerving and lane exceed indicates reduced vehicle control and hence a higher accident risk [10]. Despite these limitations standard deviation of lateral position (SDLP) is one of the most common performance metrics used, and reflects the degree of vehicular control a driver exerts in any particular driving situation. Similar measures are reflected in Time-To-Line-Crossing (TLC) factor, which is defined as the time it takes to reach the lane marking, assuming fixed steering angle and a constant speed. It is accepted, that TLC $<1 \mathrm{~s}$ implies an increased safety risk. TLC indicates that a lane exceed is likely to occur within a short time frame and therefore detects a possible risk before the lane exceed actually occurs.

\section{Summary}

There are significant differences amongst researchers concerning the choice of methods for measuring driver behaviour, mainly when it comes to assessment of the usefulness of various types of variables and their potential relation to the traffic safety. There are no straight relationships between measures and ideal condition of "traffic safety". Moreover there are no common standards for indicators and their cut-off values e.g. in different studies definition of a critical event described by the TTC value can vary from one to even few seconds. Moreover, safety is always a set of parameters and single indicators have their limitations. These variables should be calculated in relation to other indicators and general traffic conditions (number of lanes, weather, traffic density and other moderators).

On the other side traffic conflict techniques and presented commonly used measures have long history of development and include research on precise definition of different terms, data collection methods, severity measures, relationship with different types of crashes and standards for data collection. It is still a starting point to prepare a study and quantifying driver behaviour. Nevertheless presented traffic engineering variables can be found valid and reliable behavioural indicators for traffic psychologists to determine if the person is a safe driver. Future research work should be dedicated to find relations between these indicators with behaviour stated in driver questionnaires, personality and cognitive tests used for psychological assessment. These studies should be validated in the driving context. Self-reported measures are usually based on the respondent's memories and expert-report measures are taken in the naturalistic driving studies, where conditions 
are uncontrolled. However, driver's performance measures can be registered in different environments, where almost all conditions can be controlled by the experimenter. Experimenter can also manipulate with conditions and isolate relationships between factors. These measurements are widely used in driving simulators and test tracks, methods that provide safety control for the hazardous situations. It can be argued if prepared experiments are in fact real road driving experience. Expectations of respondents and presence of experimenters can influence results of the study. Nevertheless driving simulators seem to be promising tool to measure relations and define safe behaviour due to measuring different traffic indicators.

\section{References}

[1] Archer, J., Indicators for Traffic Safety Assessment and Prediction and Their Application in Micro-Simulation Modelling: A Study of Urban and Suburban Intersections, Doctoral dissertation, Royal Institute of Technology, Stockholm 2005.

[2] Chaloupka, Ch., Risser, R., Don't Wait for Accidents - Possibilities to Assess Risk in Traffic by Applying the "Wiener Fahrprobe”, Safety Science, Vol. 19, pp. 137-147, 1995.

[3] Cameron, M., Elvik, R., Nilsson's Power Model Connecting Speed and Road Trauma: Applicability by Road Type and Alternative Models for Urban Roads, Accident Analysis and Prevention, Vol. 42, pp. 1908-1915, 2010.

[4] De Winter, J. C., Dodou, D., The Driver Behaviour Questionnaire as a Predictor of Accidents: A Meta-Analysis. Journal of Safety Science, Vol. 41, pp. 463-470, 2010.

[5] Haglund, M., Aberg, L., Stability in Drivers' Speed Choice, Transportation Research Part F, Vol. 5, pp. 177-188, 2002.

[6] Highway Capacity Manual, Transportation Research Board, USA 2010.

[7] Ho, C., Spence, C., Multisensory Driver, Human Factors in Road and Rail Transport, pp. 1-35, Ashgate 2008.

[8] Hyden, C. The Development of a Method for Traffic Safety Evaluation: The Swedish Traffic Conflicts Technique, Bulletin 70, Lund University, Lund, Sweden 1987.

[9] Kloeden, C. N., McLean, A. J., Moore, V. M., Ponte, G., Travelling Speed and the Risk of Crash Involvement on Rural Roads RARU, Adelaide University, Australia 2001.

[10] Martens, M., Brouwer, R., Linking Behavioural Indicators to Safety: What is Safe and What is Not?, Proceedings of the $3^{\text {rd }}$ International Conference on Road Safety and Simulation, pp. 114, Indianapolis, USA 2011.

[11] Muhlrad, N., Traffic Conflict Techniques and Other Forms of Behavioural Analysis, Proceedings of the $6^{\text {th }}$ ICTCT Workshop, Salzburg 1993.

[12] National Highway Traffic Safety Administration, National Survey of Speeding and Unsafe Driving Attitudes and Behaviour, Washington, DC 2002.

[13] Pollatschek, M. A., Polus, A., Livneh, M., A Decision Model for Gap-Acceptance and Capacity at Intersections, Transportation Research Part B, Vol. 36, pp. 649-663, 2000.

[14] Porter, B., Berry, T., A Nationwide Survey of Red-Light Running: Measuring Driver Behaviours for the Stop Red Light Running Program, Norfolk 1999.

[15] Reason, J. Human Error, Cambridge University Press, 1990.

[16] Reason, J. T., Manstead, A., Stradling, S., Baxter, J., Campbell, K., Errors and Violations on the Roads: A Real Distinction?, Ergonomics, Vol. 33, pp. 1315-1332, 1990.

[17] Sommer, M., Herle, M., Hausler, J., Risser, R., Schutzhofer, B., Chaloupka, C., Cognitive and Personality Determinants of Fitness to Drive, Transportation Research Part F, Vol. 11, pp. 362-375, 2008.

[18] Svensson, Å., A Method for Analysing the Traffic Process, Safety Perspective, Bulletin 166, Lund University, Lund, Sweden 1998.

[19] Underwood, G., Crundall, D., Chapman, P., Driving Simulator Validation with Hazard Perception, Transportation Research Part F, Vol. 14, pp. 435-446, 2011. 
[20] Várhelyi, A., Drivers Speed Behaviour at a Zebra Crossing: A Case Study, Accident Analysis \& Prevention, Vol. 30, No. 6, pp. 731-743, 1998.

[21] Wahlberg, A. E., The Relation of Acceleration Force to Traffic Accident Frequency: A Pilot Study, Transportation Research Part F, Vol. 3, pp. 29-38, 2000.

[22] World Health Organization, Global Status Report on Road Safety - Time for Action, Geneva, Switzerland 2009. 